\title{
REFLECTION OF WATER WAVES FROM A VERTICAL VORTEX SHEET IN WATER OF FINITE DEPTH
}

\author{
W. D. MCKEE $^{1}$ AND F. TESORIERO ${ }^{2}$
}

(Received 27 March 1986; revised 20 June 1986)

\begin{abstract}
The reflection-transmission properties of water waves obliquely incident upon a vortex sheet in water of finite depth are studied. The problem is reduced to that of solving two integral equations. An accurate Galerkin solution is obtained which supports the use of the "variational method" in water wave problems that has recently been questioned by Kirby and Dalrymple.
\end{abstract}

\section{Introduction}

Even within the framework of linear theory, the study of the propagation of water waves across a horizontally sheared current has proved to be remarkably difficult. The problem is basically non-separable and shares many features and difficulties in common with the problem of propagation over varying bottom topography. A good introduction to both of these is given by Mei [3] while Peregrine [6] gives a thorough discussion of the whole matter of wave-current interactions. Two basic lines of approach have been found to be fruitful, based upon the ratio of wavelength to current scale length. The first assumes this ratio to be small and leads to approximations of $W K B$ type. Phenomena such as caustics can be incorporated into these models. The second approach takes the opposite viewpoint and represents the current by regions of constant velocity separated by one or more vortex sheets. While the first case is the one of greater oceanographical relevance, there are some coastal regions such as near river discharges or strong tidal currents where the second approach might be of more significance.

\footnotetext{
${ }^{1}$ School of Mathematics, University of New South Wales, P. O. Box 1, Kensington, N.S.W. 2033, Australia.

24 Wackett Street, Maroubra, N.S.W. 2035, Australia.

(1) Copyright Australian Mathematical Society 1987, Serial-fee code 0334-2700/87
} 
In this paper we shall consider the propagation of waves across a single vortex sheet in water of finite depth. The primary aim is to determine the reflection and transmission coefficients. The shallow-water-theory solution of this problem has been presented by Peregrine [6] (see also Mollo-Christensen [5]). The case of infinite depth was treated by Evans [1] while Smith [7] gave an approximate method of attack in water of finite depth. Recently, Tesoriero [8] 'extended Evans' work to the finite depth case. His results agreed well with those of Evans when the waves on both sides of the vortex sheet were short and with shallow water theory when the waves on both sides of the vortex sheet were long. Both Evans and Tesoriero reduced the problem to that of solving a pair of integral equations. They both used simple two-term Galerkin approximations, following the lead set by Miles [4] who studied propagation over a step change in depth. These types of solutions are generally referred to as "variational solutions" since the individual elements of the scattering matrix (see Section 3) each satisfy a variational principle and so ought to be able to be accurately approximated by simple means. These variational solutions are widely used but have recently been called into question by Kirby and Dalrymple [2]. The agreement between the results of Tesoriero for short waves and those of Evans is encouraging but not conclusive because both used the same sort of Galerkin approximation.

It is the aim of this paper to test the validity of Tesoriero's solution by including more terms in the Galerkin approximation. We find that his solutions are generally quite good, thus lending support to the variational method in this context, and then go on to present accurate results for the reflection and transmission coefficients. The problem itself is formulated in Section 2 and some of its general properties discussed. Section 3 presents the method of solution used and the results are presented and discussed in Section 4.

\section{Formulation}

We consider the propagation of small-amplitude surface gravity waves on water of constant density and depth. Axes are chosen so that the undisturbed free surface is the $x-z$ plane with the $y$-axis pointing vertically down. The ocean bottom is at $y=H$. In the absence of wave motion, there are uniform current velocities $U_{1} \mathbf{k}$ and $U_{2} \mathbf{k}$ in $x>0$ and $x<0$ respectively. Throughout this paper we will use a subscript 1 to denote the region $x>0$ and a subscript 2 to denote the region $x<0$. A wave of frequency $\omega$ is assumed to be obliquely incident from $x=+\infty$. Thus, if $\Phi_{m}$ is the velocity potential in region $m$,

$$
\Phi_{m}=U_{m} z+\phi_{m}(x, y) e^{i(p z-\omega t)} ; \quad m=1,2,
$$

where, without loss of generality, $\omega>0$ and $p>0$. 
It is convenient to non-dimensionalise lengths with respect to the depth $H$, wavenumbers with respect to $H^{-1}$, velocities with respect to $g \omega^{-1}$ (the phase speed of waves of frequency $\omega$ in still water of infinite depth) and velocity potentials with respect to $a \omega H$, where $a$ is a typical surface amplitude associated with the incident wave. The dimensionless problem for the wave velocity potential $\phi_{m}$ is then given by:

$$
\left.\begin{array}{l}
\frac{\partial^{2} \phi_{m}}{\partial x^{2}}+\frac{\partial^{2} \phi_{m}}{\partial y^{2}}-q^{2} \phi_{m}=0 \quad \text { for } 0<y<1, \\
\frac{\partial \phi_{m}}{\partial y}+K_{m} \phi_{m}=0 \quad \text { at } y=0, \\
\frac{\partial \phi_{m}}{\partial y}=0 \quad \text { at } y=1,
\end{array}\right\} \quad m=1,2
$$

with the matching conditions

$$
\left.\begin{array}{l}
\alpha_{1} \phi_{1}=\alpha_{2} \phi_{2} \\
\alpha_{2} \frac{\partial \phi_{1}}{\partial x}=\alpha_{1} \frac{\partial \phi_{2}}{\partial x}
\end{array}\right\} \text { at } x=0 \text { for } 0<y<1 .
$$

The first matching condition represents the fact that the pressure is continuous across the vortex sheet. The second is the kinematic condition implying that a particle remains on the sheet. Derivations may be found in Evans [1].

In these equations $q=p H$ and

$$
\alpha_{m}^{2}=K_{m}=Q\left(1-q \beta_{m} / Q\right)^{2},
$$

where $Q=\omega^{2} H / g$ and $\beta_{m}=\omega U_{m} / g$ is the dimensionless current velocity in region $m$.

The general solution in region $m$ may be obtained via separation of variables and, following Evans [1], will be written as

$\phi_{m}(x, y)=\operatorname{sgn} x\left\{\left(A_{m} e^{-i l_{m}|x|}+B_{m} e^{i l_{m}|x|}\right) \chi_{m}(y)+\sum_{n=1}^{\infty} C_{m n} e^{-\sigma_{m n}|x|} \psi_{m n}(y)\right\}$,

where

$$
\begin{gathered}
\chi_{m}(y)=\hat{A}_{m} \cosh R_{m}(y-1), \\
\psi_{m n}(y)=\hat{B}_{m n} \cos k_{m n}(y-1), \\
\hat{A}_{m}=2 R_{m}^{1 / 2} /\left(2 R_{m}+\sinh 2 R_{m}\right)^{1 / 2},
\end{gathered}
$$

and

$$
\hat{B}_{m n}=2 k_{m n}^{1 / 2} /\left(2 k_{m n}+\sin 2 k_{m n}\right)^{1 / 2} .
$$

In these expressions, $R_{m}$ is the unique positive solution of

$$
R_{m} \tanh R_{m}=K_{m},
$$


$k_{m n}$ is the $n$th positive solution of

$$
k_{m n} \tan k_{m n}=-K_{m}
$$

and

$$
l_{m}=\left(R_{m}^{2}-q^{2}\right)^{1 / 2}, \quad \sigma_{m n}=\left(k_{m n}^{2}+q^{2}\right)^{1 / 2} .
$$

If $l_{m}^{2}<0$, we naturally take only the solution which decays away from the vortex sheet at $x=0$. In region $m$, the set

$$
\left\{x_{m}(y), \psi_{m n}(y) ; n=1,2,3, \ldots\right\}
$$

forms a complete orthonormal set with respect to the weight function 1 . The function $\chi_{m}(y)$ is the eigenfunction for the surface wave mode while the functions $\psi_{m n}(y)$ are the eigenfunctions for the evanescent modes trapped to the vortex sheet. If $l_{m}^{2}>0$, we may define $\theta_{m}$ by

$$
l_{m}=R_{m} \cos \theta_{m}, \quad\left(0<\theta_{m}<\pi / 2\right)
$$

and so

$$
q=R_{m} \sin \theta_{m} .
$$

Thus far, the formulation has been quite general. The specific case to be treated here is that of a wave incident from $x=+\infty$ with $U_{1}=0$. Thus $\beta_{1}=0$ and we specify the angle of incidence $\theta_{1}, Q\left(=K_{1}\right.$ now $)$ and the dimensionless current strength $\beta_{2}$ in region 2 . These enable $R_{m}$ and $k_{m n}$ to be found numerically from which $l_{2}^{2}$ and all other necessary quantities are easily found. If $l_{2}^{2}>0$ we find the angle of transmission $\theta_{2}$ from

$$
q=R_{1} \sin \theta_{1}=R_{2} \sin \theta_{2} \text {. }
$$

With $\beta_{1}=0$, the $x$-component of the group velocity of a wave component $\exp \left[i\left(l_{2} x+q z-\omega t\right)\right]$ in region 2 is readily found to be

$$
c_{g_{x}}=\frac{l_{2}\left(\tanh R_{2}+R_{2} \operatorname{sech}^{2} R_{2}\right)}{2 R_{2}\left(1-q \beta_{2} / K_{1}\right)} .
$$

Thus if $1-q \beta_{2} / K_{1}>0$, which means that $\omega-p U_{2}>0$ in dimensional variables, and we take $l_{2}>0$, the transmitted wave in region 2 is represented by $\exp \left(-i l_{2} x\right)=\exp \left(i l_{2}|x|\right)$ in (3) as expected. However if $1-q \beta_{2} / K_{1}<0$ we must take $\exp \left(i l_{2} x\right)$ as being the transmitted wave in order that the group velocity be directed towards $x=-\infty$. These two cases will be referred to as "normal" and "anomalous" respectively.

One may also derive from (1), (2) and (3) the wave action flux conservation law in the form

$$
l_{1}\left\{\left|B_{1}\right|^{2}-\left|A_{1}\right|^{2}\right\}=-l_{2}\left\{\left|B_{2}\right|^{2}-\left|A_{2}\right|^{2}\right\}
$$


by using the two-dimensional form of Green's symmetrical identity. If $l_{2}^{2}<0$, all solutions are decaying at $x=-\infty$ and the right side of (4) is replaced by zero. This implies, as expected, than the incident wave is reflected with unchanged amplitude in this case.

In the normal regime, $A_{1} \exp \left(-i l_{1}|x|\right)$ represents the incident wave, $B_{1} \exp \left(i l_{1}|x|\right)$ the reflected wave, $B_{2} \exp \left(i l_{2}|x|\right)$ the transmitted wave and $A_{2}=0$ by the radiation condition. The magnitudes $R$ and $T$ of the reflection and transmission coefficients for free-surface amplitude are then found from (4) to be connected by

$$
R^{2}+T^{2}\left\{\frac{K_{1} \cos \theta_{2} \cosh ^{2} R_{1}\left(2 R_{2}+\sinh 2 R_{2}\right)}{K_{2} \cos \theta_{1} \cosh ^{2} R_{2}\left(2 R_{1}+\sinh 2 R_{1}\right)}\right\}=1,
$$

from which it follows that $R^{2}<1$, a situation referred to as "under-reflection". Setting $R=0$ in (5) gives a value for $T$ identical with that predicted by "slowly varying" $W K B$ theory which completely neglects the reflected wave. There is no paradox in this: both the $W K B$ and present theories conserve wave action flux and so setting $R=0$ in (5) must give the $W K B$ transmission coefficient.

In the anomalous regime, group velocity considerations outlined above require us to take $B_{2}=0$ and $A_{2} \exp \left(-i l_{2}|x|\right)$ to be the transmitted wave. In this case, $T^{2}$ in (5) is replaced by $-T^{2}$ and hence $R^{2}>1$, a situation referred to as "over-reflection". In the special case $\omega-p U_{2}=-\omega$, it follows that $K_{2}=K_{1}$ and hence that $R_{2}=R_{1}$. The surface wave eigenfunctions on each side of the vortex sheet are then identical and no evanescent modes are needed to satisfy the matching conditions (2) at $x=0$. However we readily find from these conditions that $R$ and $T$ are both infinite. This is sometimes called "resonant over-reflection". For the infinite depth case, Evans [1] noted the equality of the surface wave eigenfunctions when $\omega-p U_{2}=-\omega$. However, he incorrectly identified $B_{2} \exp \left(i l_{2}|x|\right)$ as the transmitted wave and put $A_{2}=0$. In both the finite and infinite depth cases, this leads to the conclusion that $R=0$ and $T=1$, i.e. perfect transmission with no reflection. Group velocity considerations also show the assertion in his paper that $R$ and $T$, expressed as functions of $\beta_{2}$, are symmetric about $\beta_{2}=\operatorname{cosec} \theta_{1}$ to be incorrect.

In reality, there will always be a shear layer of finite thickness rather than a vortex sheet. This implies that the waves will always encounter a critical layer where $\omega-p U_{2}=0$ before entering the over-reflective regime. The relationship between the actual reflection and transmission coefficients in this case and those calculated assuming a vortex sheet is a subject worthy of further investigation. 


\section{The integral equations}

Following the lines set out by Evans [1], one may readily reduce our problem to that of solving for $0<y<1$ the integral equation

$$
\sum_{m=1}^{2} \alpha_{m}\left(A_{m}+B_{m}\right) \chi_{m}(y)=\int_{0}^{1} G(y, t) \mathscr{U}(t) d t
$$

where

$$
G(y, t)=G(t, y)=\sum_{m=1}^{2} \sum_{n=1}^{\infty} K_{m} \sigma_{m n}^{-1} \psi_{m n}(y) \psi_{m n}(t)
$$

and

$$
\mathscr{U}(y)=\alpha_{1}^{-1} \mathscr{U}_{1}(y)=\alpha_{2}^{-1} \mathscr{U}_{2}(y) \quad(\text { by }(2)),
$$

where

$$
\mathscr{U}_{m}(y)=\partial \phi_{m} /\left.\partial x\right|_{x=0} .
$$

The only essential difference between our problem and that of Evans is that, in the kernel $G(y, t)$ of the integral equation, his integral over the continuous spectrum of evanescent modes in the infinite depth case is here replaced by an infinite sum over the discrete spectrum of evanescent modes which occurs in the finite depth case.

The problem may further be reduced to that of solving for $0<y<1$ the pair of integral equations

$$
d_{m}(y)=\int_{0}^{1} G(y, t) u_{m}(t) d t ; \quad m=1,2,
$$

where

$$
d_{m}(y)=\alpha_{m} \chi_{m}(y)
$$

Once this has been done, we find the so-called scattering matrix $S$ from

$$
S_{m n}=\int_{0}^{1} d_{m}(t) u_{n}(t) d t ; \quad(m, n)=1,2 .
$$

The wave velocity potential amplitudes on either side of the vortex sheet are then connected by

$$
\left(B_{1} B_{2}\right)^{T}=\mathscr{T}\left(A_{1} A_{2}\right)^{T}
$$

where

$$
\mathscr{T}=(\mathscr{L}+i S)^{-1}(\mathscr{L}-i S)
$$


and

$$
\mathscr{L}=\left[\begin{array}{cc}
l_{1} & 0 \\
0 & l_{2}
\end{array}\right]
$$

From these, the (complex) reflection and transmission coefficients for free surface height may readily be found.

Thus, the problem ultimately reduces to that of solving (6). Tesoriero [8] sought an approximate 2-term Galerkin solution of the form

$$
u_{m}(t) \simeq \sum_{s=1}^{2} w_{m s} d_{s}(t) ; \quad m=1,2 .
$$

His results for $R$ and $T$ agreed quite well with shallow water theory when $K_{1}$ and $K_{2}$ were both small and with those of Evans [1] when they were both large. As mentioned earlier, this type of "variational solution" was introduced into the water-wave literature by Miles [4] and has been widely used every since. The solution of Evans [1] is of this kind. That author also applied the variational method to a different problem for which an exact solution was available. Agreement was generally good. More recently, however, Kirby and Dalrymple [2] have cast doubts on the accuracy of the variational method when applied to propagation over a trench by comparing its predictions with those obtained by other means.

It is one purpose of this paper to investigate the accuracy of (7) in this context by including more terms in the approximation. Specifically, we take $N$ eigenfunctions from each side and so seek an approximate $2 \mathrm{~N}$-term Galerkin solution of the form

$$
u_{m}(t) \simeq \sum_{s=1}^{2 N} w_{m s} d_{s}(t), \quad m=1,2
$$

where

$$
d_{j}(t)=\alpha_{j} \chi_{j}(t) \text { for } j=1,2
$$

and

$$
\left.\begin{array}{l}
d_{2 j+1}(t)=\alpha_{1} \psi_{1 j}(t) \\
d_{2 j+2}(t)=\alpha_{2} \psi_{2 j}(t)
\end{array}\right\} \quad j=1,2, \ldots, N-1 .
$$

Substituting this into (6), multiplying by $d_{n}(y)$ and integrating from 0 to 1 gives

$$
F=W E \text {, }
$$

where the $2 \times 2 N$ matrix $F$ is given by

$$
f_{m n}=\int_{0}^{1} d_{m}(y) d_{n}(y) d y
$$


and the $2 \dot{N} \times 2 N$ symmetric matrix $E$ by

$$
e_{n s}=\int_{0}^{1} \int_{0}^{1} G(y, t) d_{n}(y) d_{s}(t) d y d t .
$$

Once the equations (9) have been solved for $W$, the scattering matrix is found from

$$
S_{m n}=\sum_{s=1}^{2 N} w_{n s} f_{m s}
$$

From this, the reflection and transmission coefficients are found as outlined above.

The approximate solution employed here preserves the symmetry of the scattering matrix and the wave action flux conservation law becomes an identity in the elements of $S$; i.e. to within the limits of round-off error, wave action flux is conserved by this approximation. All the integrals involved in the $F$ and $E$ matrices are elementary. However, some care is needed for short waves since, for example, terms such as $\sinh 2 R_{m}$ which occur in the normalization constants $\hat{A}_{m}$ can overflow for large $R_{m}$. A careful examination shows that the $\hat{A}_{m}$ are only ever needed in the combinations $\hat{A}_{m} \sinh R_{m}$ and $\hat{A}_{m} \cosh R_{m}$. For large $R_{m}$, say 50 or larger, these may both be replaced by the asymptotic form $\left(2 R_{m}\right)^{1 / 2}$. Evaluation of $R$ and $T$ at $R_{m}=50$ using both forms showed no differences. In this and similar ways, the problem of overflow was avoided.

\section{Results and discussion}

The numerical procedure outlined above was implemented on a VAX 11/780 at Melbourne University. Using double precision FORTRAN, this gives about 16 decimal digits. The linear equations (9) were solved using the IMSL subroutine LEQ1S. The number of terms used in the kernel of the integral equations (6) was increased until the method had converged. Typically, 80 terms were needed but fewer sufficed for smaller values of $\beta_{2}$. In fact, sufficient accuracy for the drawing of graphs could generally be obtained with about 20 terms. There is also the question of the number of terms to use in the Galerkin approximation (8). One might naively expect that more terms would lead to greater accuracy. While this is generally true for small $N$, there is a practical limit imposed by the fact that the $2 N \times 2 N$ matrix $E$ becomes more and more ill-conditioned as $N$ increases.

It has been pointed out by a referee that this kind of behaviour is only to be expected from the general properties of Fredholm equations of the first kind and that there is usually an optimum number of terms at which the $L_{2}$-norm of the error in (6) is minimised. Typically, $N=5$ was about as far as we could go on our 
machine before the effects of the ill-conditioning became serious. However, as we shall see, this is generally more than enough terms for accurate results to be obtained.

We begin by presenting a typical example with $K_{1}=3$ and $\theta_{1}=45^{\circ}$. In Figures 1,2 and 3 we show the amplitudes of the transmission and reflection coefficients as well as the phase of the reflection coefficient for $-3 \leqslant \beta_{2} \leqslant \beta_{c}$ where $\beta_{c}$ is the value of $\beta_{2}$ at which $l_{2}=0$, i.e. $\theta_{2}=90^{\circ}$. Beyond this value $\left(\beta_{c}=0.2376 \ldots\right.$ for these particular parameters) the waves are totally reflected from the vortex sheet until, for very large $\beta_{2}$, wave propagation is again possible in the over-reflective regime. The phase of the transmission coefficient has not been shown since it is very close to zero-never more than $5^{\circ}$ in magnitude. In plotting the reflection coefficient we have adopted the sign convention advocated by Smith [7]; namely to associate a negative sign with the magnitude (and make the appropriate change of $180^{\circ}$ to the phase) for $\beta_{2}<0$. For relatively short waves at least, this makes the phase of the reflection coefficient continuous as $\beta_{2}$ passes through zero and removes the cusp in the magnitude at $\beta_{2}=0$. These three figures show the results of the two-term, four-term and eight-term Galerkin solutions (8). The six-term results are indistinguishable from the eight-term ones. In Figures 1 and 2 we have also shown the results of the averaging method of

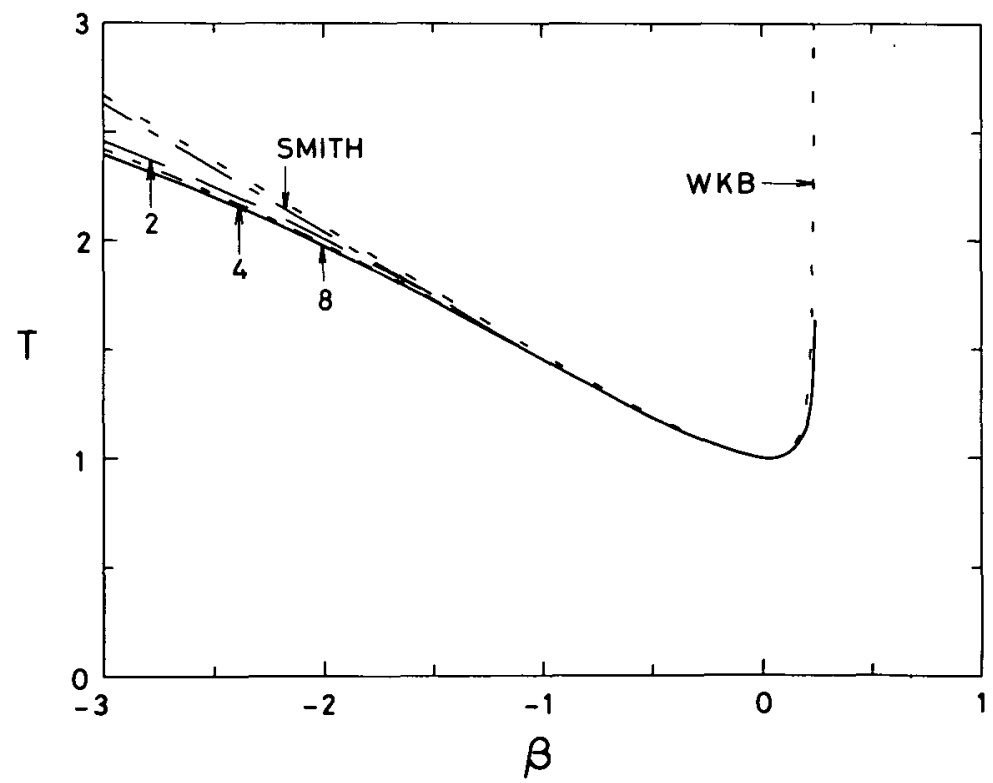

Figure 1. Transmission coefficient magnitude for $K_{1}=3$ and $\theta_{1}=45^{\circ}$. Shown are the results of the 2-term (- - - , 4-term (- - - $)$ and 8-term (- $\longrightarrow$ Galerkin solutions as well as those of Smith's averaging method (__ - - ) and the WKB method (- $\left.-{ }_{-}\right)$. 


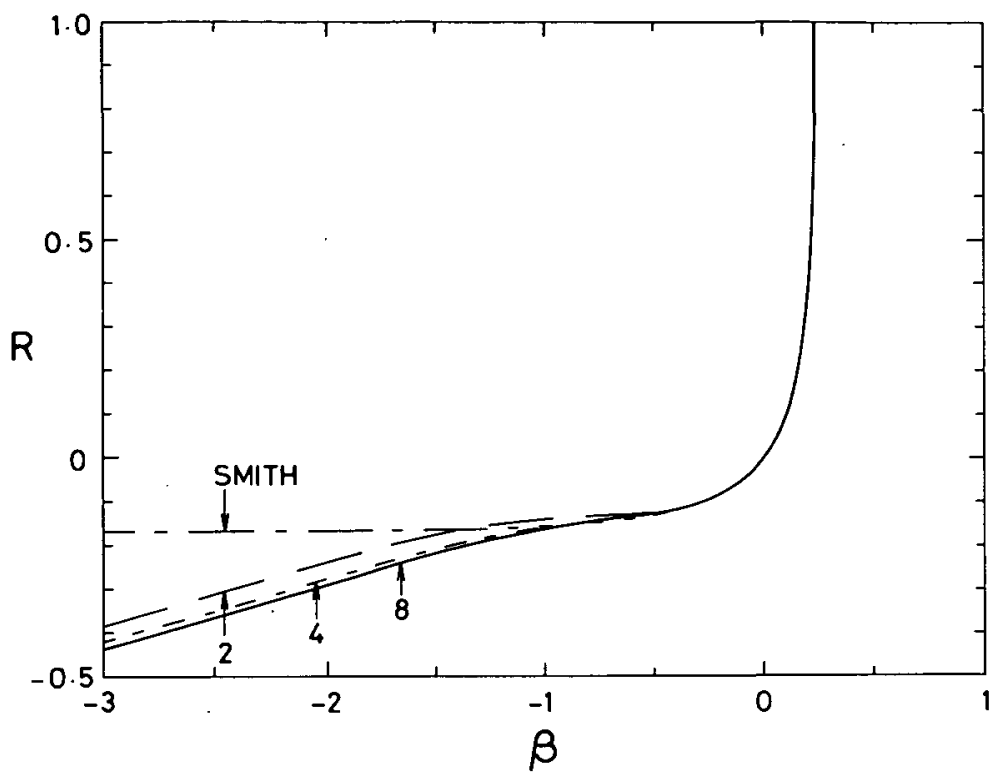

Figure 2. As for Figure 1 except that the magnitude of the reflection coefficient is shown. This is defined as having the same sign as the dimensionless current $\beta_{2}$. There is no $W K B$ solution.

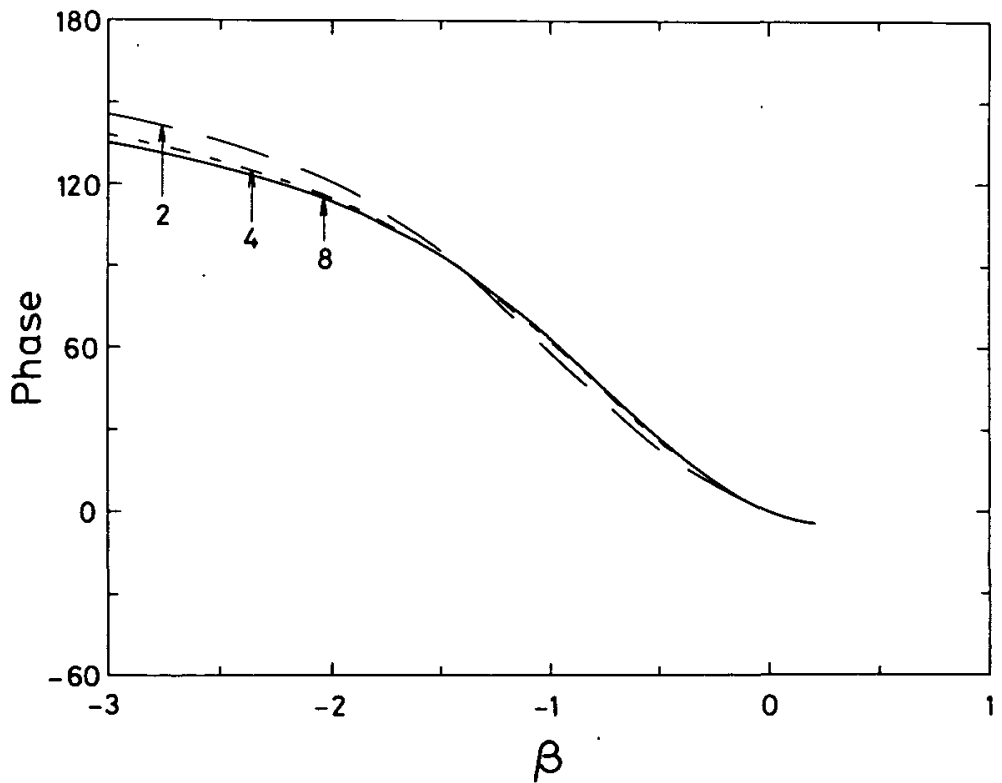

Figure 3. As for Figure 2, showing the phase in degrees of the reflection coefficient. Smith's method gives no information on this quantity. 
Smith [7], adapted to water of finite depth, and, in Figure 1, the $W K B$ transmission coefficient amplitude is also shown. Neither of these methods gives any phase information nor considers the influence of the evanescent modes.

The results depicted in Figures 1,2 and 3 are broadly typical of all cases we have investigated; namely that the two-term Galerkin solution, which is in fact that of Tesoriero [8], is really quite adequate for most purposes especially for small values of $\beta_{2}$. This conclusion supports the use of the "variational method" as applied to this particular problem: good results are obtained from simple approximations. It should also be noted that only small values of $\beta_{2}$ are of any physical relevance since $\beta_{2}=\omega U_{2} / g \simeq 0.64 U_{2} / \tau$ if $U_{2}$ is measured in meters per second and $\tau$ is the wave period in seconds.

We now proceed to investigate the effects of finite depth on the reflection and transmission coefficients. In Figures 4 and 5 we show the reflection and transmission coefficients for various values of $K_{1}$ (i.e. for various depths if we fix the wave frequency) for an angle of incidence $\theta_{1}=30^{\circ}$. Figures 6 and 7 show the same things for $\theta_{1}=60^{\circ}$. In all these Figures, we used six terms in the Galerkin solution (8); the eight-term results being virtually indistinguishable. From these it is seen that for strong opposing currents the magnitudes of the reflection and transmission coefficients are decreasing functions of $K_{1}$, i.e., the shallower the

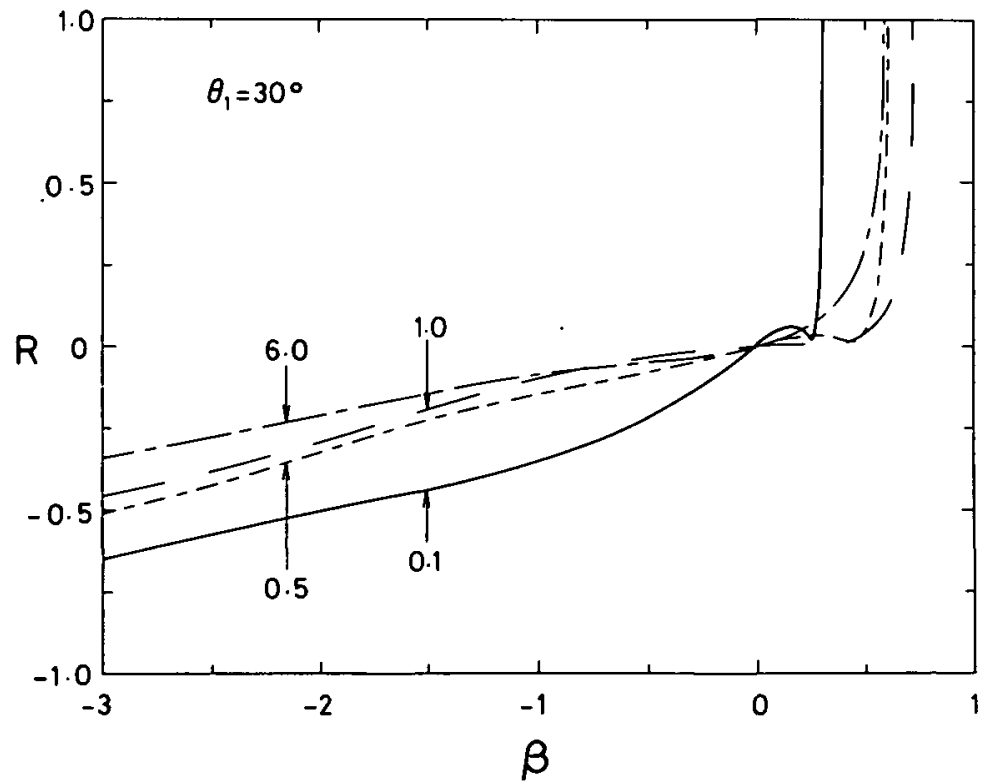

Figure 4. Reflection coefficient magnitude when $\theta_{1}=30^{\circ}$ for $K_{1}=0.1(\longrightarrow), K_{1}=0.5$ $(---\longrightarrow), K_{1}=1.0(\longrightarrow-\longrightarrow)$ and $K_{1}=6.0(--\longrightarrow) .1$ 


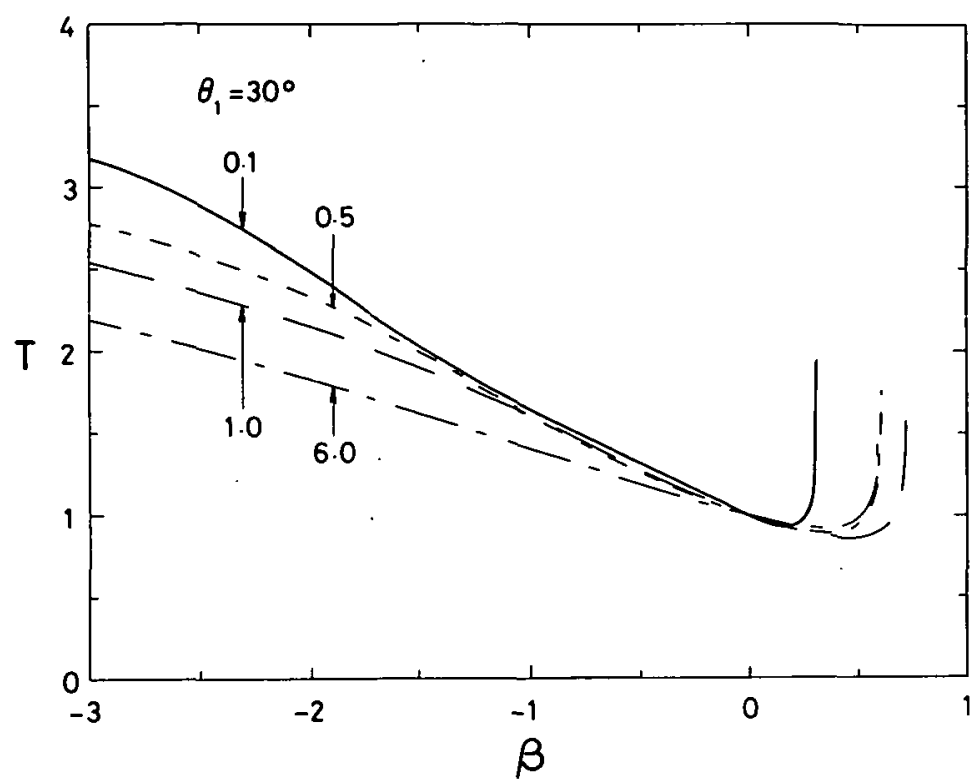

Figure 5. As for Figure 4, showing the transmission coefficient magnitude.

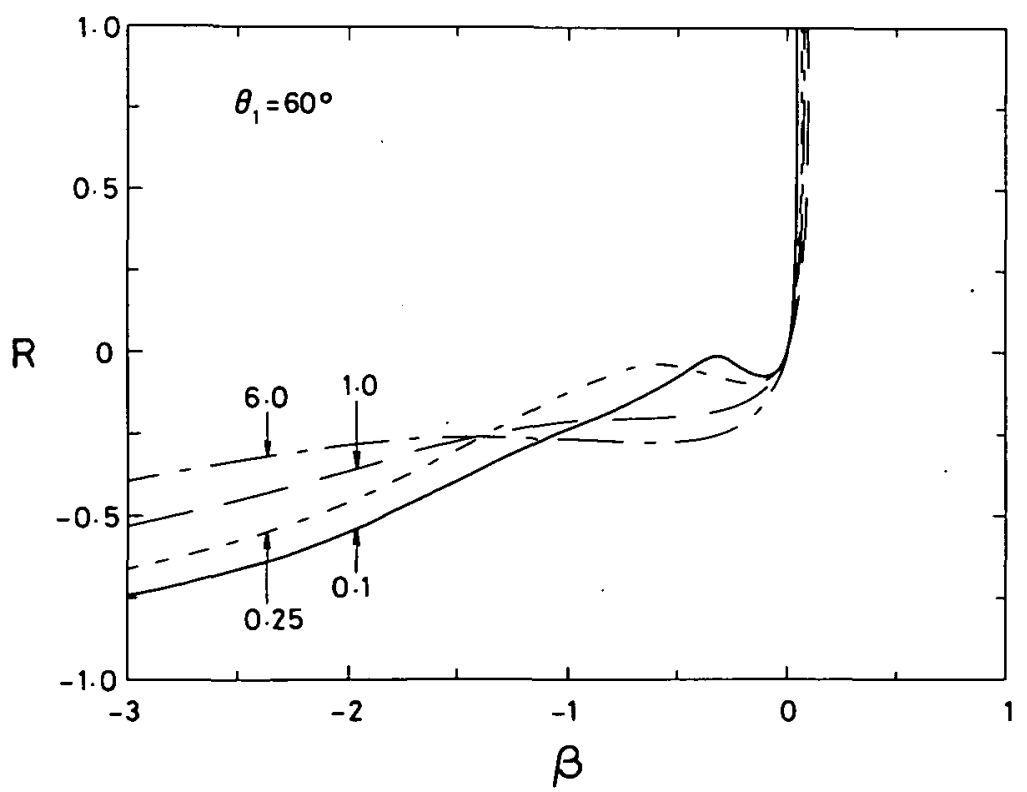

Figure 6. As for Figure 4 with $\theta_{1}=60^{\circ}$ and $(----)$ now represents $K_{1}=0.25$. 
water, the larger they are. For weak opposing currents and for following currents this is seen to be not necessarily true. Indeed the critical value of $\beta_{2}$ at which $R \rightarrow 1$ is not even a monotone function of $K_{1}$. Shallow water theory predicts that $R=0$ at $\beta_{2}=K_{1}^{1 / 2}\left[\operatorname{cosec} \theta_{1}-\sec \theta_{1}\right]$ as well as at $\beta_{2}=0$. This may be deduced from expressions given in [5]. For small but finite $K_{1}$, our computations indicate that the magnitude of $R$ has a minimum near this value of $\beta_{2}$. This is clearly seen in Figures 4 and 6 for $K_{1}=0.1$. Interestingly, a minimum still occurs, but is less dramatic, for larger values of $K_{1}$.

We close by presenting some results in the over-reflective regime: Figures 8 and 9 show the reflections and transmission coefficients when $\theta_{1}=45^{\circ}$ for $K_{1}=0.1$ and $K_{1}=4.0$. Only the over-reflective values are shown and eight Galerkin terms were used. The curious features evident near $\beta_{2}=2.5$ when $K_{1}=0.1$ and near $\beta_{2}=3.5$ when $K_{1}=4.0$ are quite genuine. They also occur when 2,4 or 6 Galerkin terms are used. Indeed, there is very little difference between the results obtained with $2,4,6$ or 8 Galerkin terms.

In summary, our results bridge the gap between the shallow water results of Peregrine [6] and the deep water results of Evans [1]. They also lend support to the use of the variational method in water wave problems involving discontinuities.

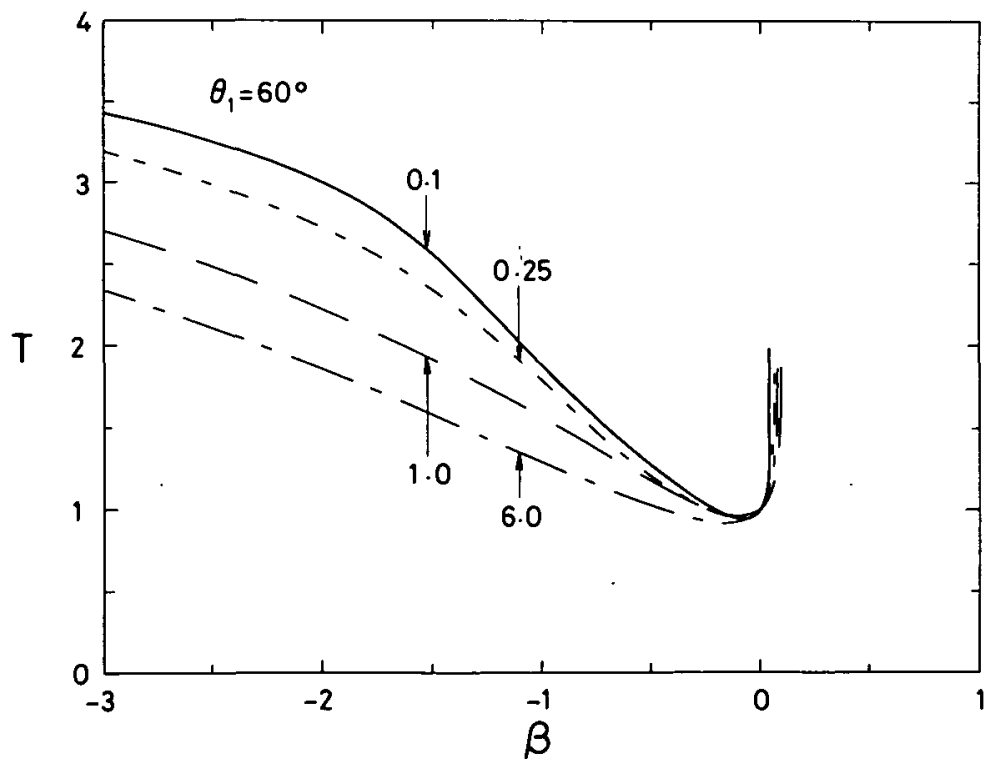

Figure 7. As for Figure 5 with $\theta_{1}=60^{\circ}$ and (- - - ) again representing $K_{1}=0.25$. 


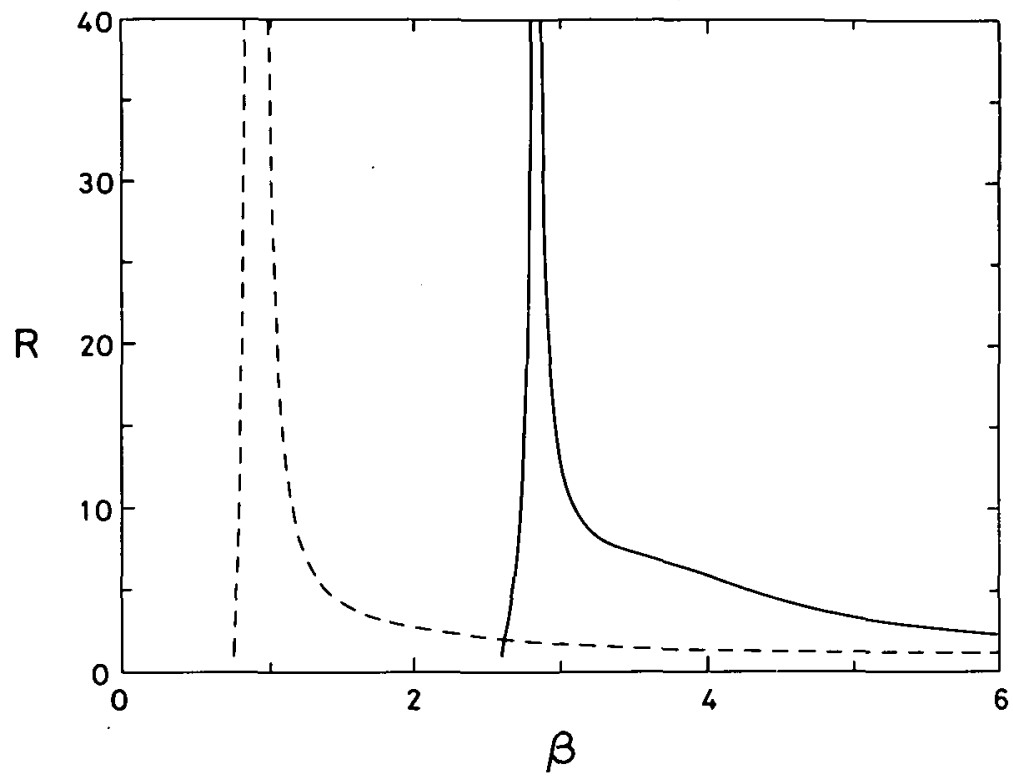

Figure 8. The reflection coefficient magnitude as a function of $\beta_{2}$ when $\theta_{1}=45^{\circ}$ in the over-reflective regime when $K_{1}=0.1(---)$ and $K_{1}=4.0(-)$. Only the results for the over-refleetive regimes (which start at $\beta_{2}=0.7533 \cdots$ and $\beta_{2}=2.5980 \cdots$ ) are shown. Resonant over-reflection occurs when $\beta_{2}=0.8795 \cdots$ and $\beta_{2}=2.8265 \cdots$.

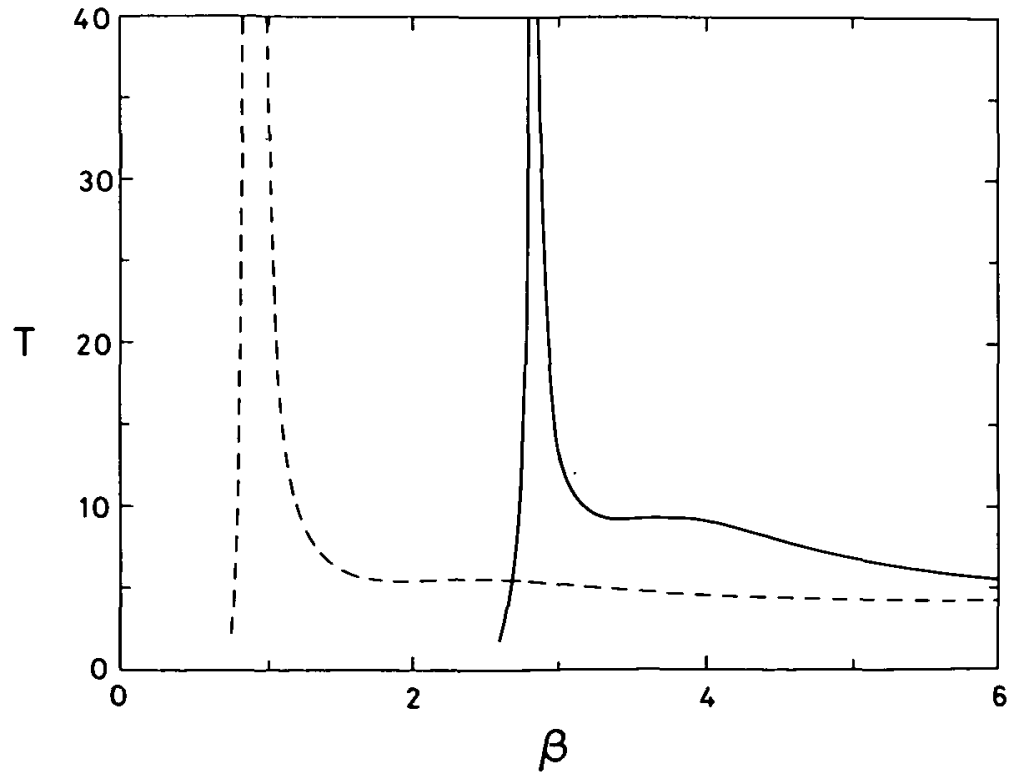

Figure 9. As for Figure 8, showing the transmission coefficient magnitude. 


\section{Acknowledgements}

All the computations were done and the manuscript written while the first author was on leave at the University of Melbourne. He wishes to thank the University of New South Wales for granting leave and the Chairman and staff of the Department of Mathematics at the University of Melbourne for their hospitablity. The comments of Roger Grimshaw and of the anonymous referee are also appreciated.

\section{References}

[1] D. V. Evans, "The transmission of deep-water waves across a vortex sheet", J. Fluid Mech. 68 (1975) 389-401.

[2] J. T. Kirby and R. A. Dalrymple, "Propagation of obliquely incident water waves across a trench", J. F/uid Mech. 133 (1983) 47-63.

[3] C.-C. Mei, The applied dynamics of ocean surface waves (Wiley, New York, 1983).

[4] J. W. Miles, "Surface-wave scattering matrix for a shelf", J. Fluid Mech. 28 (1967) 755-767.

[5] E. Mollo-Christensen, "Over-reflection of horizontally propagating gravity waves by a vertical shear layer", Phys. Fluids 21 (1978) 1908-1911.

[6] D. H. Peregrine, "Interaction of water waves and currents", Adv. in Appl. Mech. 16 (1976) 9-129.

[7] J. Smith, “On surface gravity waves crossing weak current jets”, J. Fluid Mech. 134 (1983) $277-299$.

[8] F. Tesoriero, "The propagation of surface waves across a shear current in water of finite depth", B. Sc. Thesis, School of Mathematics, University of New South Wales, 1984. 